OPEN ACCESS

Edited by:

Shihui Yang,

Hubei University, China

Reviewed by:

Yong Xu,

Nanjing Forestry University, China

Richa Arora

Lovely Professional University, India JunLi Ren,

South China University of

Technology, China

${ }^{*}$ Correspondence:

Seonghun Kim

seonghun@kribb.re.k

Specialty section:

This article was submitted to Bioenergy and Biofuels,

a section of the journal

Frontiers in Energy Research

Received: 03 April 2019

Accepted: 12 July 2019

Published: 26 July 2019

Citation:

Kim S (2019) Xylitol Production From

Byproducts Generated During

Sequential Acid-/Alkali-Pretreatment

of Empty Palm Fruit Bunch Fiber by an

Adapted Candida tropicalis.

Front. Energy Res. 7:72.

doi: 10.3389/fenrg.2019.00072

\section{Xylitol Production From Byproducts Generated During Sequential Acid-/Alkali-Pretreatment of Empty Palm Fruit Bunch Fiber by an Adapted Candida tropicalis}

\author{
Seonghun $\mathrm{Kim}^{1,2 *}$ \\ 1 Jeonbuk Branch Institute, Korea Research Institute of Bioscience and Biotechnology (KRIBB), Jeongeup, South Korea, \\ ${ }^{2}$ Department of Biosystems and Bioengineering, KRIBB School of Biotechnology, University of Science and Technology \\ (UST), Daejeon, South Korea
}

Xylose is a pentose sugar with the potential to convert a variety of valuable chemical products. In this study, acidic pretreatment wastewater generated during a sequential acid-/alkali-pretreatment process was recycled to increase the hydrolyzed hemicellulose fraction from empty palm fruit bunch fiber (EPFBF), a lignocellulosic biomass. The xylose in the reused wastewater was subjected to overliming and an activated charcoal column was used to remove inhibitory compounds, for xylitol fermentation using the adapted C. tropicalis strain. The cell growth and xylose uptake rates in the adapted strain were 1.7- and 5-fold higher, respectively, compared to the wild-type strain. During batch fermentation using the adapted yeast strain and the post-pretreated xylose solution, 35.2 $\pm 0.8 \mathrm{~g} / \mathrm{L}$ xylitol was obtained within $61 \mathrm{~h}$ for a production yield of $0.44 \mathrm{~g}$ xylitol/g xylose. These results indicate that xylose in the byproducts produced in the bioethanol process could be recovered for production of xylitol.

Keywords: acidic pretreated biomass wastewater, sequential acid-/alkali-pretreatment process, xylose, xylitol, adapted C. tropicalis, overliming, empty palm fruit bunch fiber

\section{INTRODUCTION}

Lignocellulosic biomass including agricultural plant byproducts, forestry wood residues, and other cellulosic materials are all potential resources of low-cost fermentable sugars, which could be used as biofuels and biorefinery chemicals (Kim and Kim, 2012, 2014; Kim et al., 2013; Brethauer and Studer, 2015; Caicedo et al., 2016; Kim, 2018a). These materials are composed of three major components: cellulose, hemicellulose and lignin. These polymeric materials could be hydrolyzed by either a physicochemical or a biological process, to generate monomeric sugar compounds in addition to a variety of aromatic/aliphatic compounds (Caicedo et al., 2016). However, these polymeric structures have chemically complex and resistant structures. Therefore, for generation of fermentable sugars from lignocellulose, physical, chemical, and biological pretreatment processes have been applied to convert the complex and rigid structures of lignocellulose into fermentable sugars (Silveira et al., 2015; Capolupo and Faraco, 2016). Depending on the pretreatment process and the type of biomass, i.e., soft or hard biomass, the physicochemical properties of the pretreated biomasses could be changed to obtain the maximum yield of fermentable sugars (Silveira et al., 2015). 
Several different biomass pretreatment options exist, including physical, chemical, and biological methods. Chemical pretreatment under acid and alkali conditions, combined with high temperature and pressure or mechanical disruption, is currently the most widely used biomass pretreatment process (Shen et al., 2015; Silveira et al., 2015; Capolupo and Faraco, 2016). Among the various chemical pretreatment processes reported to date, acid and alkali pretreatments were shown to be the most effective for reducing hemicellulose or delignification in biomass under mild operating conditions (Alvira et al., 2010; Kim and Kim, 2014; Silveira et al., 2015; Kim, 2018a,b). In our previous work, we combined these two agents to pretreat empty palm fruit bunch fiber (EPFBF), which is an abundant byproduct in the palm oil industry, using a sequential acid-/alkali-pretreatment process to enhance the cellulose content per unit biomass (Kim et al., 2012; Kim and Kim, 2013).

EPFBF contains relatively high levels of hemicellulose and lignin per gram biomass compared to other lignocellulosic biomasses (Piarpuzán et al., 2011; Kim and Kim, 2012, 2013; Kim et al., 2012; Raman and Gnansounou, 2014). In the first pretreatment step, the removal of hemicellulose increased the pore sizes of the biomass (Kim et al., 2012). Therefore, it increased both the accessibility and digestibility of cellulase (Kim et al., 2012; Kim and Kim, 2013). The hydrolyzed hemicellulose fractions were solubilized in the pretreatment water. This is considered to be wastewater, containing extremely high concentrations of organic substances and low $\mathrm{pH}$ under wastewater treatment guidelines (Zhang et al., 2010; Abdolali et al., 2014). In a case study on second-generation (2G) ethanol production, wastewater treatment during the pretreatment process accounted for $\sim 20 \%$ of the total production cost (Humbird et al., 2011). Therefore a reduction of the costs associated with wastewater treatment would decrease the overall costs of using lignocellulosic materials (Humbird et al., 2011; Kim et al., 2019).

Nevertheless, acid extracted during EPFBF pretreatment under the sequential acid-/alkali-pretreatment process could be a valuable resource due to its high xylose content. Moreover, acidic wastewater produced during the first step of pretreatment still contains acidic catalysts. Thus, the chemical catalysts could be reused and enriched in the hydrolyzed hemicellulose fraction of the wastewater. In the case of EPFBF, the chemically hydrolyzed hemicellulose mostly comprised xylose, which is a pentose sugar that can be fermented to produce xylitol, xylonate, and other biorefinery products (Kim et al., 2012, 2019; Toivari et al., 2012; Brethauer and Studer, 2015). Moreover, xylitol is an expensive sugar alcohol and one of top 12 high value-added intermediate chemicals as a building block for organic synthesis (Werpy et al., 2004; Ravella et al., 2012). The downstream process for purification and formulation have been already established well (Aliakbarian et al., 2012). The sugar alcohol production from the wastewater could be also an alternative approach for overall cost reduction of bioethanol production.

In this work, I examined the effect of recycling the acidic wastewater generated during the first biomass pretreatment step under a sequential acid-/alkali-pretreatment process. The acidic pretreated solution and biomass washing water were separated from the biomass, and then these solutions were reused five times to enrich the pentose sugar content. After the acidic treatment solution had been neutralized, the biomass pretreatment byproduct containing a high concentration of xylose, a valuable fermentable sugar, was applied for xylitol production.

\section{MATERIALS AND METHODS}

\section{Material}

The EPFBF was obtained from a local oil processing company in Malaysia. It was washed with tap water to remove soil and other particles and then dried at $105^{\circ} \mathrm{C}$ for $24 \mathrm{~h}$ (Kim, 2018b).

\section{Chemical Pretreatment}

The overall xylose extraction procedure based on sequential acid/alkali-pretreatment of EPFBF is summarized in Figure 1. Dry EPFBF (10\% w/v) with no physical treatment was soaked in diluted $\mathrm{H}_{2} \mathrm{SO}_{4}$ solution within the concentration range of $0.1 \sim 8.0 \%(\mathrm{v} / \mathrm{v})$ and heated in an autoclave at $121^{\circ} \mathrm{C}, 15 \mathrm{psi}$ for $15 \mathrm{~min}$. During the first pretreatment step, the dilute acidtreated EPFBF was obtained from the acid solution, soaked in the same volume of water, and occasionally mixed for $1 \mathrm{~h}$. The washed pretreated-EPFBF was dried at $105^{\circ} \mathrm{C}$ for $24 \mathrm{~h}$. During the second pretreatment step, the dried EPFBF was treated using $10 \mathrm{~N} \mathrm{NaOH}$ solution, as described in the previous work (Kim et al., 2012). The compositions of the non-treated and pretreated biomasses were analyzed using the National Renewable Energy Laboratory (NREL) chemical analysis and testing laboratory analytical procedures (LAPs) of the US Department of Energy (DOE): the lignin contents in biomass were analyzed by LAP-003 and LAP-004.

\section{Recycling Acidic Wastewater for Biomass Pretreatment}

The acidic wastewater generated during the sequential acid/alkali-pretreatment was reused in a repeat of the first acid pretreatment process. The biomass-soaked sulfuric acid solution and washing water were used separately for the acid pretreatment and biomass washing, respectively. During the acidic-thermal pretreatment, the acidic solution was evaporated and the ratio of biomass to solution was fixed at $10 \%(\mathrm{w} / \mathrm{v})$. The solution was recycled five times during the first biomass pretreatment step. This increased the acid-hydrolyzed sugar concentration in the solution. Monosaccharides and other sugar derivatives were analyzed after each biomass pretreatment step.

\section{Analysis of Carbohydrate Content in Wastewater Generated From the Acidic Pretreatment Processes}

To determine the carbohydrate content of the acidic wastewater generated during the chemical pretreatment process, the xylose, glucose and other sugars in the wastewater samples were diluted with distilled water, without any further neutralization, and then passed through a $0.20-\mu \mathrm{m}$ filter. The monosaccharides hydrolyzed from the pretreated biomass in the acidic wastewater samples were then identified and quantified using high-performance liquid chromatography (HPLC), as described below. 


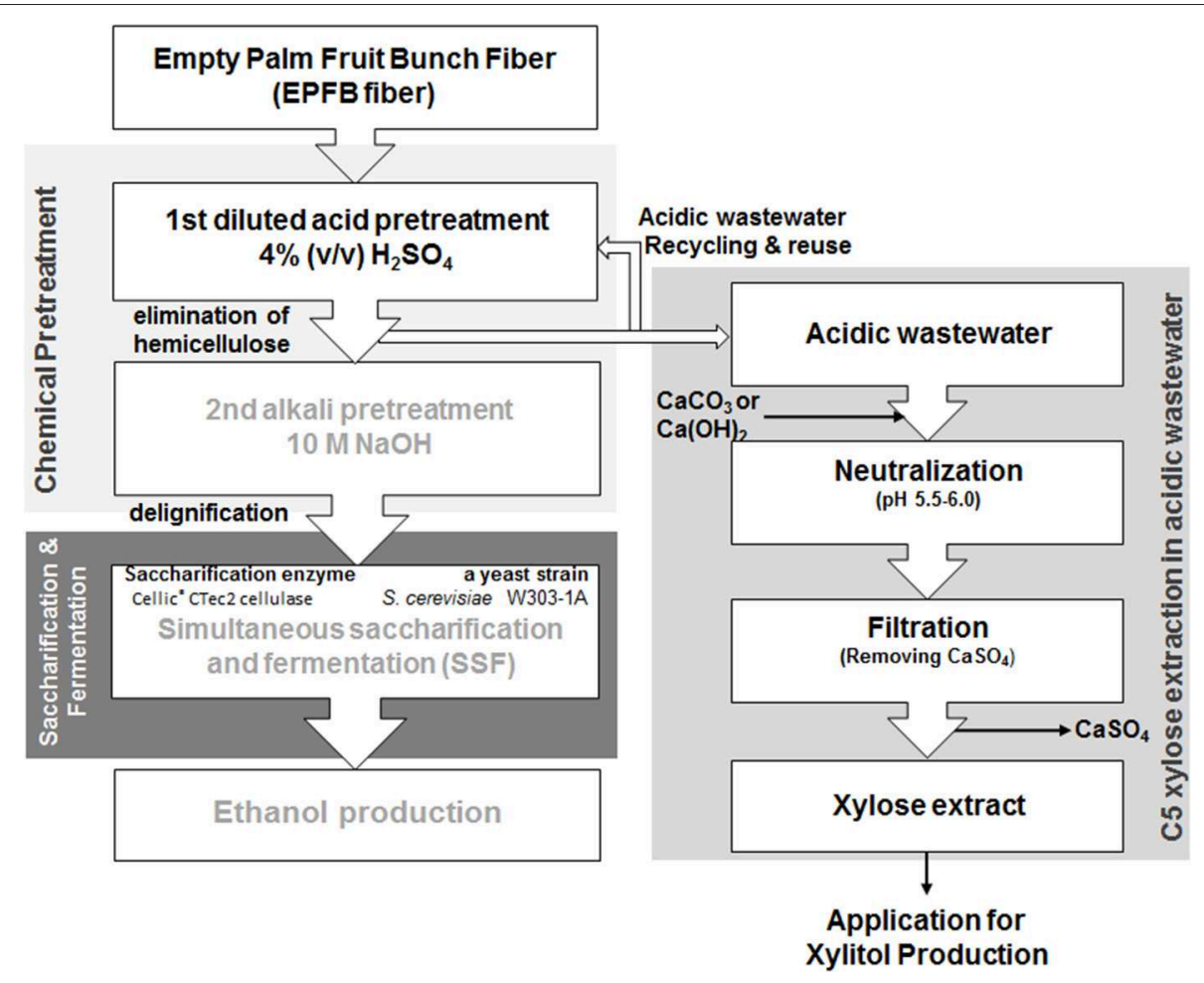

FIGURE 1 | The overall procedure for xylose extraction from the acidic wastewater byproducts generated during the first step of the sequential acid-/alkali-pretreatment process, for utilization of empty palm fruit bunch fiber (EPFBF).

\section{Neutralization of the Acid Wastewater}

For neutralization of the acidic wastewater solution for yeast cultivation, the solution was slowly titrated with solid calcium carbonate $\left(\mathrm{CaCO}_{3}\right)$ or calcium hydroxide $\left(\mathrm{Ca}(\mathrm{OH})_{2}\right)$ to a $\mathrm{pH}$ of 5.5-6.0. The temperature of the titrated solution was maintained at around $25-35^{\circ} \mathrm{C}$ in an ice-water bath. The $\mathrm{CaSO}_{4}$ precipitate in the titrated solution was removed by centrifugation and then passed through an activated charcoal fixed-bed column. The solution was filtrated using a $0.20-\mu \mathrm{m}-$ cellulose membrane filter. The final pale, yellowish solution was stored at room temperature.

\section{Microorganism, Growth Conditions, and Cultivation}

Candida tropicalis CBS94 was used as a xylitol fermentation strain (https://www.uniprot.org/taxonomy/559300). The yeast strain was cultivated in complex medium containing $1 \%$ Bacto yeast extract, $2 \%$ Bacto peptone, and $2 \%(\mathrm{v} / \mathrm{v})$ xylose at $30^{\circ} \mathrm{C}$ and $200 \mathrm{rpm}$ for $24 \mathrm{~h}$, for seed culture and maintenance.

\section{Yeast Strain Adaptation}

The single yeast strain colony was transferred into $25 \mathrm{~mL}$ of liquid broth containing $10 \%(\mathrm{v} / \mathrm{v})$ of neutralized biomass hydrolysate, in turn containing $25 \mathrm{~g} / \mathrm{L}$ xylose and a $6.7 \mathrm{~g} / \mathrm{L}$ yeast nitrogen base. After a $24-\mathrm{h}$ incubation at $30^{\circ} \mathrm{C}, 1 \%(\mathrm{v} / \mathrm{v})$ the pre-culture was transferred to the fresh culture broth and then cultivated under the equal condition. This process was repeated 10 times. In each batch culture, cell growth, xylose, and xylitol content were monitored. After strain adaptation, the final culture was spread on an agar medium having the same culture composition, and single colonies were selected for xylitol fermentation.

\section{Xylitol Fermentation}

For xylitol fermentation, the neutralized acidic wastewater solution containing $\sim 80 \mathrm{~g} / \mathrm{L}$ xylose was re-filtrated through a $0.22-\mu \mathrm{m}$ pore filter membrane system (Corning, Corning, NY, USA). The seed-culture of the adapted $C$. tropicalis strain $10 \%(\mathrm{v} / \mathrm{v})$ was then inoculated into a 1-L fermentor (FMT ST$\mathrm{S}$; Fermentec, Cheongju, South Korea) with a $0.5 \mathrm{~L}$ working volume containing the filtered xylose solution and a $1 \%(\mathrm{w} / \mathrm{v})$ yeast nitrogen base. The fermentor was operated at $30^{\circ} \mathrm{C}$ with stirring at $240 \mathrm{rpm} / \mathrm{min}$. The xylitol fermentation was performed in triplicate.

\section{Analytical Procedures}

Levels of monosaccharide sugars and organic acids were determined using an HPLC system equipped with a refractive index detector, an auto-sampler, and an Aminex HPX-87P column (Bio-Rad, Hercules, CA, USA) for monosaccharide analysis, or with a Rezex ROA-Organic Acid H+ column (Phenomenex Inc., Torrance, CA, USA) for organic acid analysis, as described previously (Kim, 2018a,b). All samples were clarified by filtration with a $0.20-\mu \mathrm{m}$ filter and then injected into 
the analytical HPLC column. All analyses were performed in triplicate.

\section{RESULTS}

\section{Characterization of Xylose Extract in the Acidic Wastewater}

In my previous work, the cellulose, hemicellulose, and lignin content of the raw EPFBF material before chemical pretreatment was measured to be $39.8,17.3$, and $28.8 \%$, respectively (Kim et al., 2012). The relative composition of these three components varied depending on the harvest time, cultivation area, and several environmental factors associated with plant growth. It has been reported that dried EPFBF consists of $41.3-46.5 \%$ cellulose, 25.3-32.5\% hemicellulose, and 27.6-32.5\% lignin (Kim, 2018b). During the sequential acid-/alkali-pretreatment process, the hemicellulose content in the biomass was reduced during the first diluted acid pretreatment step. During the thermalacidic pretreatment step, the concentration of the dilute sulfuric acid increased from 0 to $8 \%(\mathrm{v} / \mathrm{v})$, while the hemicellulose content decreased from 17.3 to $1.6 \mathrm{~g}$ per $100 \mathrm{~g}$ of biomass (Kim et al., 2012).

The hemicellulose portion in the pretreated biomass was extracted and hydrolyzed in the diluted acidic pretreatment solution. After the first pretreatment, xylose, a monomer sugar of xylan, which is a major component of hemicellulose, was mainly observed in the acidic wastewater (Figure 2A). The major component in the acidic solution was xylose depolymerized from hemicellulose (82\%). Minor compounds in the solution included oligosaccharides (6\%), disaccharides (4\%), glucose (4\%), acetate (3\%), and other unidentified substances (1\%). Increasing the strength of the diluted sulfuric acid from 0 to $8 \%(\mathrm{v} / \mathrm{v})$ caused the xylose concentration to increase from $0.69 \pm 0.2$ to $33.8 \pm 0.5$ $\mathrm{g} / \mathrm{L}$ (Figure 2B). The xylose concentration in the solution tended to increase in proportion to the elimination of hemicellulose extracted from the biomass. Up to $4 \%(\mathrm{v} / \mathrm{v})$ diluted sulfuric acid, the xylose concentration was dramatically enhanced. However, the efficiency of the xylose extraction was only slightly enhanced by using a stronger acidic solution of $8 \%(\mathrm{v} / \mathrm{v})$. Thus, these data suggest that $4 \%(\mathrm{v} / \mathrm{v})$ diluted sulfuric acid could be the optimum concentration for hemicellulose extraction under these pretreatment conditions $\left(121^{\circ} \mathrm{C}, 15 \mathrm{psi}\right.$, for $\left.1 \mathrm{~h}\right)$.

\section{Effect of the Number of Acidic Wastewater Reuse Cycles Used in Biomass Pretreatment}

The acidic wastewater from the first pretreatment was dark brown-colored and contained the acidic substances extracted from the biomass. However, the solution still contained the sulfuric acidic catalyst, which could be reused in further biomass pretreatment steps. To test the effect of recycling acidic wastewater on the hemicellulose fraction extraction, the solution was reused for biomass pretreatment of fresh, untreated EPFBFs. After the pretreatment, the level of sugars and their related compounds, dissolved in both the generated acidic solution and biomass washing water, were analyzed (Figure 3).

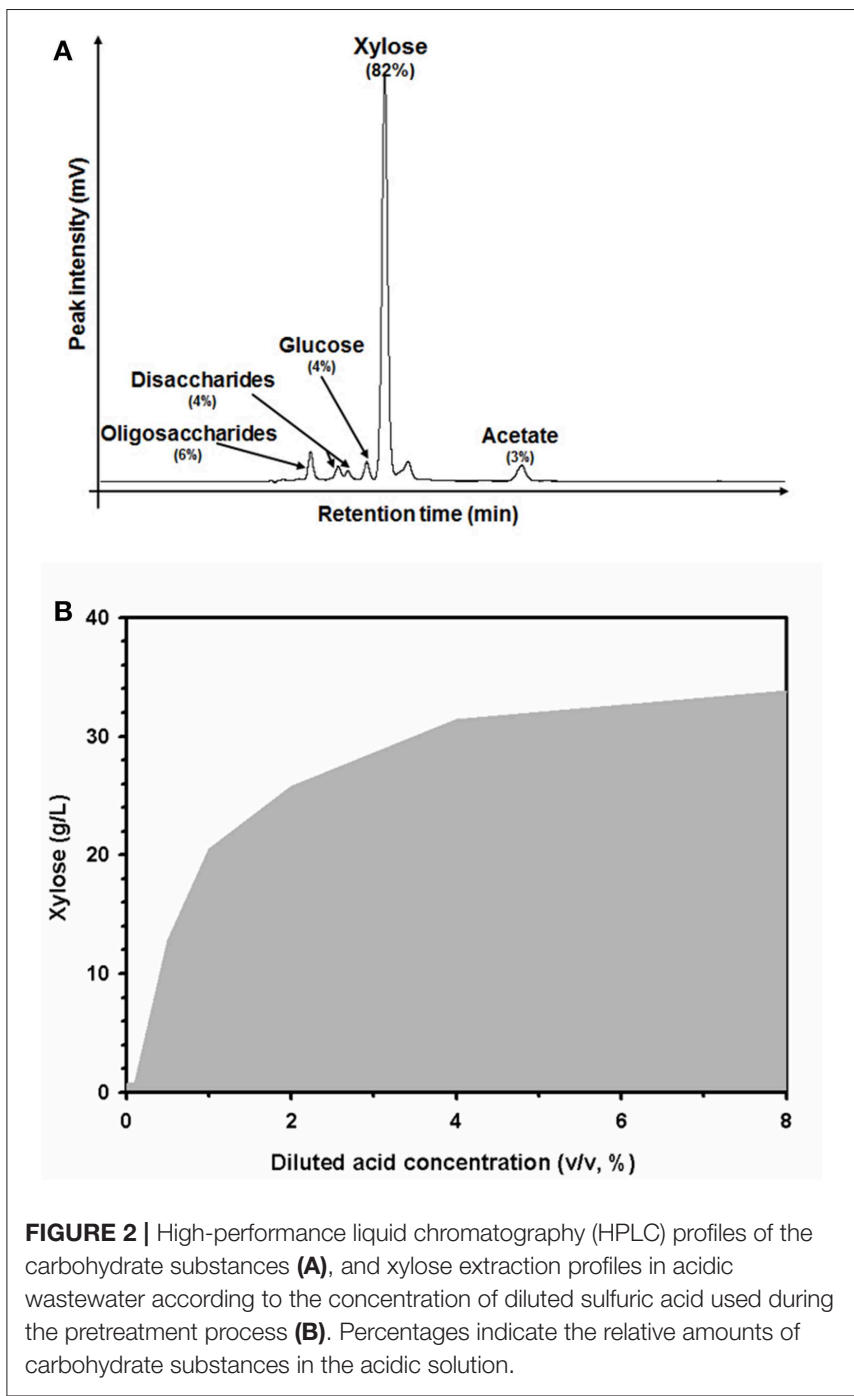

In agreement with previous studies, xylose was found to be the most abundant sugar compound in both the acidic solution and the washing water (Figure 3A). In both solutions, the xylose concentration was relatively similar. The xylose concentration (hydrolyzed from the hemicellulose fraction) reached $82.3 \pm$ $0.5 \mathrm{~g} / \mathrm{L}$ in the acidic solution after five cycles. On average, the concentration of xylose increased by 1.74 -fold with each pretreatment, which is in agreement with first-order reaction kinetic theory (data not shown). Interestingly, the biomass washing solution contained a relatively large amount of xylose $(38.8 \mathrm{~g} / \mathrm{L})$ after five washing cycles. Acetate, glucose, and furfural were additionally detected in both solutions as minor compounds (Figure 3B). The concentration amounts were in the order of acetate $>$ glucose $>$ furfural in each pretreatment batch. Interestingly, the concentration of acetate was relatively high, where this compound is hydrolyzed from acyl groups attached to hemicellulose. In addition, the levels of furfural, a thermally degraded pentose compound, increased after batch pretreatment. Small amounts of xylose were converted into furfural under 

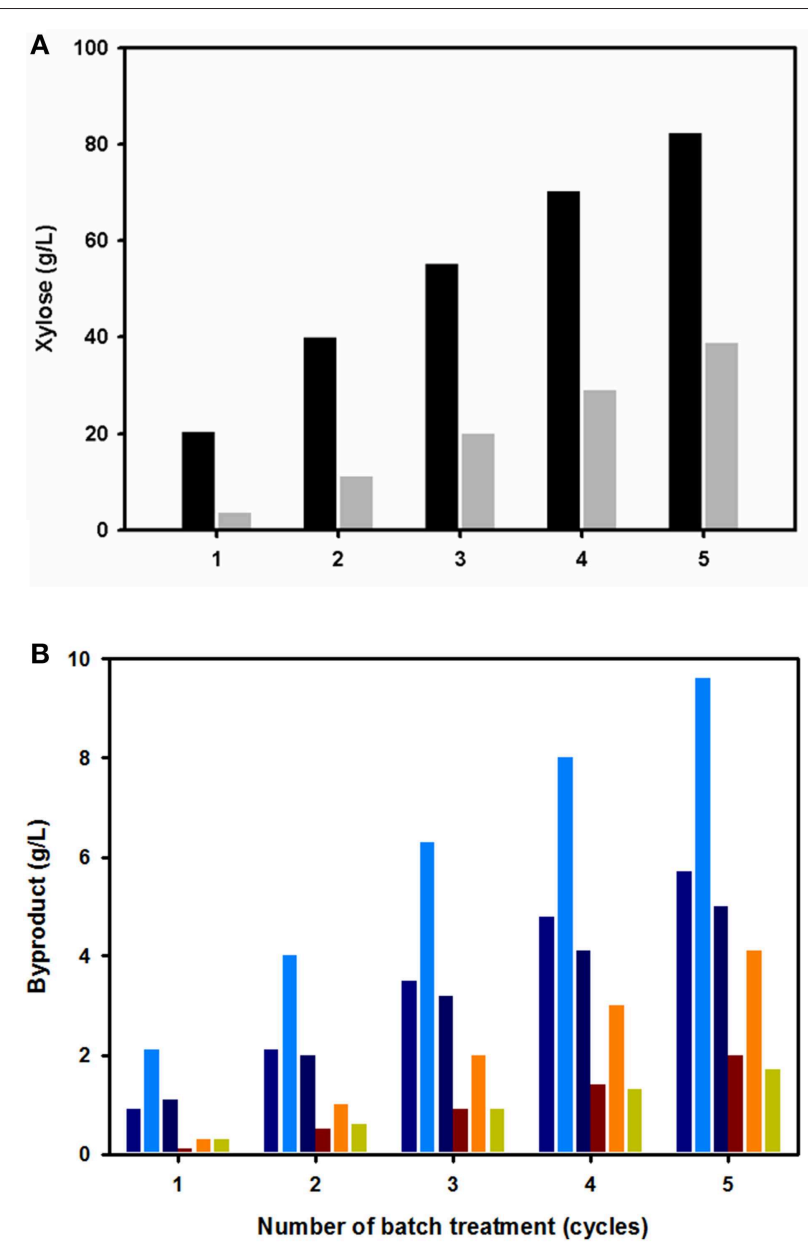

FIGURE 3 | Effect of the number of diluted acid batch pretreatments on the concentrations of extracted soluble substances in the acidic wastewater (black bars) and washing solution (gray bars). Xylose, the most abundant sugar substance (A), and other minor soluble byproducts [glucose (blue-violet), acetate (blue), and furfural (deep blue)] (B) remained in the acidic wastewater. The minor soluble compounds [glucose (brown), acetate (orange), and furfural (yellow-green)] in the washing solution.

the thermal acidic pretreatment conditions. Glucose was also present as a hydrolyzed cellulose fraction during the diluted acid pretreatment process.

\section{Preparation of Xylose Solution From the Acid Wastewater}

To apply xylose solution for xylitol fermentation, the acidic solution was titrated to $\mathrm{pH}$ 5.5-6.0 with solid $\mathrm{CaCO}_{3}$ or $\mathrm{Ca}(\mathrm{OH})_{2}$. Sulfuric acid present in the acid wastewater containing xylose was precipitated by the formation of calcium sulfate $\left(\mathrm{CaSO}_{4}\right)$. After centrifugation and filtration to remove the calcium salt, the clarified xylose solution with a deep brown color was passed through an activated charcoal (mesh 100 particle size) fixed-bed column to reduce the levels of thermally degraded sugar products, unknown aliphatic and aromatic compounds, and thermally degraded lignin products. The xylose solution prepared from the acidic pretreatment wastewater had a pale yellow color. Following path though the column, the total volume of the xylose solution decreased $\sim 5 \%$. The concentrations of xylose, glucose, and acetate were $80.1 \pm 1.2,5.1 \pm 0.5$, and 4.2 $\pm 0.3 \mathrm{~g} / \mathrm{L}$, respectively. The final prepared xylose solution did not contain any residual furfural (data not shown).

\section{Adaptation of the Yeast Strain to Xylose Extract}

To produce xylitol via fermentation using the xylose solution, a yeast strain know as Candida tropicalis CBS94 was chosen. The wild-type strain grew slowly in the xylose solution prepared from the acidic wastewater due to unknown growth inhibitors (data not shown). Thus, the strains were adapted to $2.5 \%(\mathrm{w} / \mathrm{v})$ xylose extract solution; for the adaptation, a single colony was inoculated in the minimal medium containing xylose solution and cultivated for $24 \mathrm{~h}$. The $1 \%(\mathrm{v} / \mathrm{v})$ batch culture was then transferred to fresh medium; this process was repeated 10 times. The cell growth, xylose uptake, and xylitol production were all monitored (Figure 4). During the first batch culture, cell growth (Figure 4A) was very slow due to the low level of xylose uptake (Figure 4B). Xylitol did not accumulate in the culture medium (Figure 4B).

After the first cultivation, cell growth and xylose utilization rates gradually increased with longer adaptation times. Repeating the batch culture phase 10 times gave a total adaption time of $240 \mathrm{~h}$, which enhanced cell growth without any lag phase. Comparing the initial status of the yeast cells, xylose consumption and xylitol production were dramatically increased in the adapted strain. After the 10 repeated cycles of batch culture, the adapted yeast strain had completely consumed the xylose and produced $9.62 \pm 0.25 \mathrm{~g} / \mathrm{L}$ xylitol in $24 \mathrm{~h}$ (Figure 4B). The cell growth and xylose uptake rates in the adapted strain were 1.7 - and 5 -fold higher, respectively, vs. the values for the wild-type yeast.

\section{Production of Xylitol by the Adapted Yeast Strain}

To assess xylitol production using the xylose solution prepared from the wastewater of the acidic pretreatment, batch fermentation was performed with the adapted $C$. tropicalis strain in a $1-\mathrm{L}$ jar fermentor. The initial concentration of xylose in the culture medium supplemented with a yeast nitrogen base was $80.0 \pm 1.5 \mathrm{~g} / \mathrm{L}$. After inoculation of the adapted yeast strain, xylitol fermentation proceeded gradually for $61 \mathrm{~h}$ (Figure 5). Yeast cell growth was slow until $9 \mathrm{~h}$, according to a lag phase, and then increased exponentially before entering a stationary phase between 45 and $58 \mathrm{~h}$ (Figure 5). The concentration of xylose decreased gradually due to cell growth. All of the xylose in the culture broth was depleted at $54 \mathrm{~h}$. The highest xylitol concentration was $35.2 \pm 0.8 \mathrm{~g} / \mathrm{L}$ at $61 \mathrm{~h}$, giving a xylitol yield of $0.44 \mathrm{~g}$ xylitol/g xylose at $61 \mathrm{~h}$.

Ultimately, the fermentable xylose in the culture broth was completely used up for xylitol fermentation by the adapted yeast. No reduction in xylitol concentration was observed until the xylose had been depleted. Xylitol fermentation of the xylose 

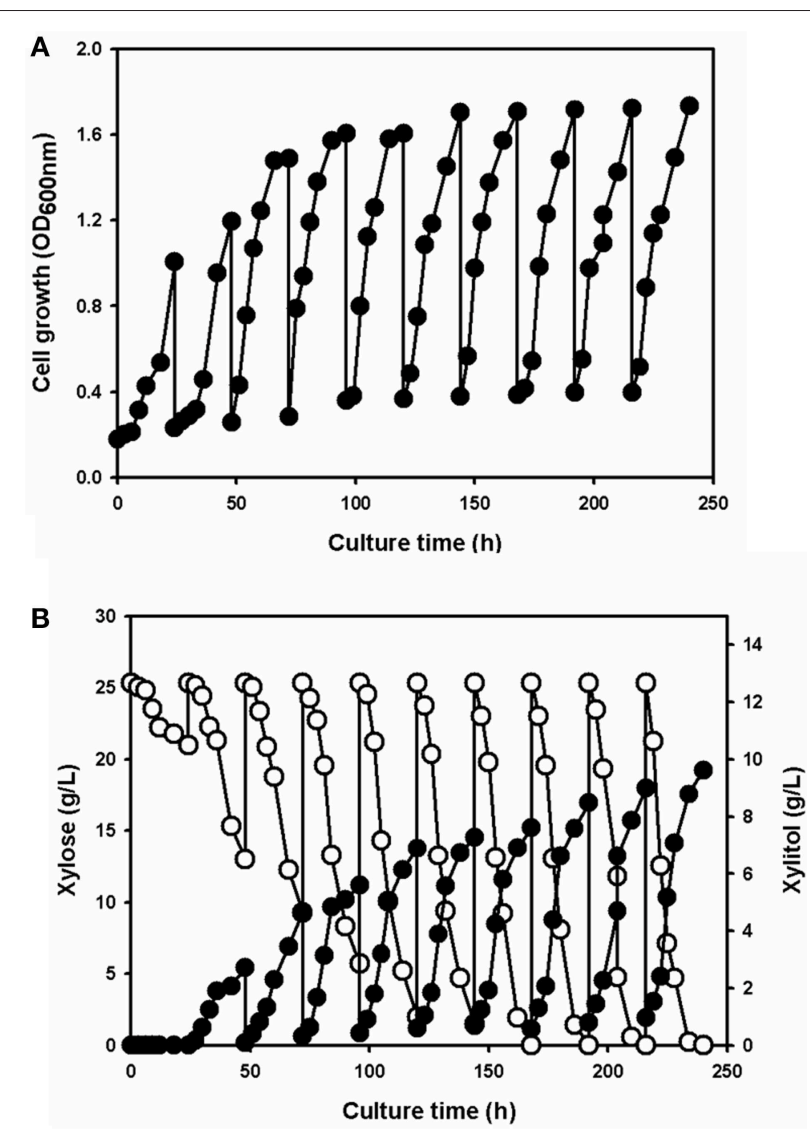

FIGURE 4 | The profiles of cell growth (A), xylose consumption (open circle) and xylitol production (filled circle) (B) for Candida tropicalis strain CBS94 adapted in the batch culture containing the culture medium supplemented with xylose extract as the sole carbon source.

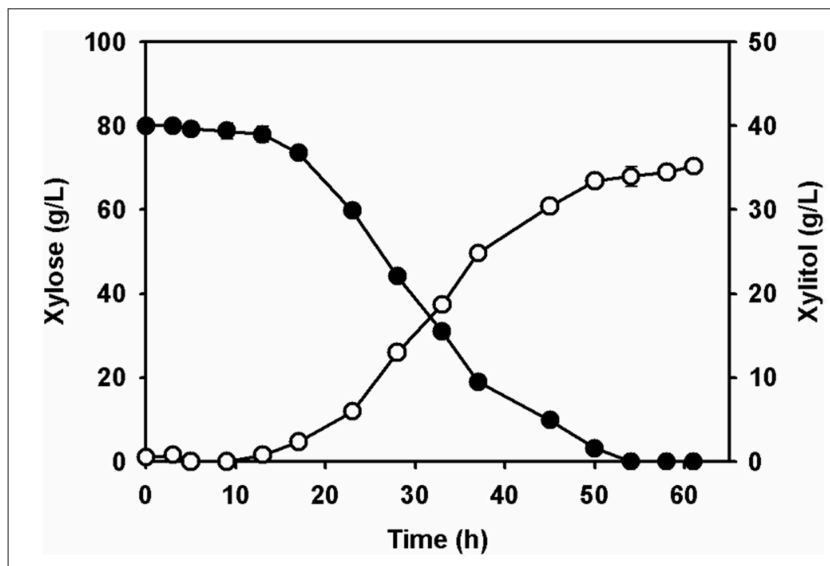

FIGURE 5 | Batch fermentation of the xylose extract by the adapted Candida tropicalis strain CBS94 in a 1.1-jar fermenter. Profiles of xylitol (open circle) and xylose (filled circle) in fermenter were analyzed by HPLC.

solution prepared from the acidic pretreatment wastewater by the adapted C. tropicalis produced several metabolites, including acetic acid, ethanol, and glycerol (data not shown). All of the metabolites produced by the yeast were present at concentrations $>1 \mathrm{~g} / \mathrm{L}$, lower than that of the xylitol. In addition, these metabolites accumulated, with no consumption occurring in the fermentation broth. These metabolites did not appear to affect cell growth or xylitol productivity during the fermentation process. Levels of these byproduct metabolites were probably too low to have any negative effects on cell metabolism due to the short xylitol fermentation time.

\section{DISCUSSION}

Sequential acid-/alkali-pretreatment of EPFBF with dilute sulfuric acid followed by sodium hydroxide is among the chemical pretreatment technologies used to enhance cellulose content in pretreated biomass, by removing hemicellulose and lignin (Kim et al., 2012). It is also effective in reducing the size of fibers and the strength of physical fibril interactions, thus enhancing the efficiency of the enzymatic process. The first pretreatment process using the diluted sulfuric acid effectively removed the hemicellulose fraction from the biomass. After this process, the acidic pretreatment wastewater was found to have a high concentration of xylose, which was hydrolyzed from hemicellulose. In this study, xylose and other substances in the acidic wastewater generated during EPFBF pretreatment were analyzed, and the pentose sugar enriched in the reused wastewater was applied for xylitol fermentation using an adapted yeast strain.

In addition, the acidic pretreatment solution still harbored sulfuric acid as an acidic catalyst, which could potentially be reused during further pretreatment cycles. The acidic pretreatment wastewater and biomass washing water were reused five times for the repeated batch pretreatment. The hemicellulose removal efficiency in the biomass pretreatment did not decrease with the sequential acid-/alkali-pretreatment (data not shown). Pretreatment of wastewater generated during bioethanol production is considered to be a cost-intensive treatment process (Zhang et al., 2010; Abdolali et al., 2014).

Reusing acidic wastewater could reduce the processing cost of the chemical catalyst and acid wastewater treatment. In addition to the reuse of pretreatment wastewater, fermentable sugar compounds chemically hydrolyzed from hemicellulose in the biomass could serve as resources in future biorefinery applications. The diluted sulfuric acid pretreatmenthydrolyzed hemicellulose fraction in EPFBF mainly comprised monomerized xylose (Figure 2A). Other pentose sugars such, as arabinose, fructose, and so on were not detected in the acidic solution extracted from EPFBF by diluted sulfuric acid pretreatment. The repeated batch pretreatment also enriched the xylose content of the acid pretreatment solution (Figure 2B).

It is also important to consider the fact that thermal acidic pretreatments generate thermally degraded products, such as furfural (2-furaldehyde), hydroxymethylfurfural (5hydroxymethyl-2-furaldehyde, 5-HMF), and other aliphatic acids (acetic, formic, and levulinic acid) (Parajó et al., 1998; Palmqvist and Hahn-Hägerdal, 2000). During the diluted acid pretreatment of EPFBF, acetic acid and furfural were the 
main compounds detected in the acid wastewater. Moreover, the amounts of these compounds increased following reuse of the sulfuric acid for the repeated batch pretreatment (Figure 3B). To overcome the effects of these inhibitory compounds to allow utilization of the xylose sugar present in acidic wastewater, calcium salt precipitation, a charcoal column, and strain adaptation were applied in this study. Other detoxification strategies, such as physical methods (evaporation, stream stripping, solvent extraction, phase separation, membrane filtration, etc.), chemical methods (ion-exchange column), and biological methods (enzyme-mediated approaches) could also be applicable to reduce or eliminate these inhibitory chemicals (Parajó et al., 1997; Mpabanga et al., 2012).

The soluble sulfate ion in the acid wastewater was easily precipitated by calcium ion to form $\mathrm{CaSO}_{4}$ and gypsum; then, the precipitated gypsum was removed by filtration. During this process, other inhibitory compounds were co-precipitated with formatted salts; however, the xylose in the solution was lost. Therefore, calcium salt precipitation could serve as a simple and cost-effective procedure, and the remaining gypsum could be used as a soil acid neutralizer for sustainable agriculture.

After neutralization and filtration, the solution contained unknown substances generated by the acid-thermal pretreatment process and decomposition of the biomass (Parajó et al., 1997; Mpabanga et al., 2012). One way to reduce unknown inhibitory compounds is to apply an adsorption method that uses either activated charcoal (Vinke and van Bekkum, 1992; Zhang et al., 2011) or ion exchange resins (Mpabanga et al., 2012). Application of activated charcoal could be an effective method for the adsorption of furfural, 5-HMF, and phenolic compounds, despite loss of xylose in the solution. In this study, the xylose solution passing through a charcoal column was a pale yellow color due to the adsorption of colored compounds (data not shown). In a previous study, charcoal adsorption with a combination of overliming and organic solvent extraction for Eucalyptus wood hydrolysates improved both xylitol production and yeast cell growth due to reduced fermentation inhibition (Parajó et al., 1997). Other methods involving activated charcoal adsorption could be useful to improve the quality of the fermentable sugars obtained from lignocellulose hydrolysate, but the cost of post-pretreatment during the overall preparation process might need to be considered when reusing byproducts in biorefinery processes (Melin and Hurme, 2011; Jönsson and Martín, 2016; Liu et al., 2017).

Adaptive evolution using lignocellulosic hydrolysates is an alternative strategy to overcome the inhibitory byproducts that arise during pretreatment of lignocellulose under acidic conditions (Liu, 2011; Jönsson and Martín, 2016). A microorganism quickly responds to stress conditions by reprogramming metabolic pathways and regulatory machinery, and overexpressing stress response proteins, etc., to achieve homeostasis in vivo and stress tolerance (Liu, 2011; Thompson et al., 2016; de Witt et al., 2019). Candida tropicalis, a xylose fermentable yeast strain, could be responsible for the inhibitory compounds associated with xylitol production (Kim et al., 2019). Although the strain was inhibited by unknown inhibition compounds in the first-step culture in this study, the repeated batch culture improved cell growth (Figure 4A) and xylitol production (Figure 4B). However, the adaptive mechanisms remain unknown due to gaps in our understanding of the cellular processes. Nevertheless, previous metabolite analysis of C. tropicalis treated with an inhibitory compound mixture demonstrated that cell growth and xylitol production could be influenced by changing the xylose uptake and xylitol oxidation rate, as well as by metabolites accumulating according to the redox balance and osmoregulation (Wang et al., 2015). Repeated batch culture outcome might be affected by the $\mathrm{NAD}^{+} / \mathrm{NADH}^{+}$ratio, which is important in xylose reduction and xylitol oxidation. Although inhibitory compounds disturbed the redox balance in cells, in vivo detoxification mechanisms could correct any imbalance during adaptation periods. This should be evaluated further in the context of biochemical and genetic changes in the adapted strains, to achieve a stress-tolerant phenotype.

\section{CONCLUSION}

This study showed that the acidic pretreatment wastewater generated during the sequential acid-/alkali-pretreatment process was reusable, and could be employed to increase the hemicellulose fraction from EPFBF, a lignocellulosic biomass, and produce xylitol via the hydrolyzed xylose present in the post-pretreated solution. Recycling the acidic wastewater five times in the repeated biomass pretreatment resulted in the accumulation of up to $82.3 \pm 0.5 \mathrm{~g} / \mathrm{L}$ of xylose hydrolyzed from hemicellulose. The xylose in the reused wastewater was subjected to overliming and an activated charcoal column was used to remove inhibitory compounds, for xylitol fermentation using the adapted $C$. tropicalis strain. The cell growth and xylose uptake rates in the adapted strain were 1.7- and 5fold higher, respectively, compared to the wild-type strain. In batch fermentation using the adapted yeast strain with the post-pretreated xylose solution, $35.2 \pm 0.8 \mathrm{~g} / \mathrm{L}$ xylitol was obtained within $61 \mathrm{~h}$ for a production yield of $0.44 \mathrm{~g}$ xylitol/g xylose. Taken together, these results confirm that acidic pretreatment of wastewater could be used to generate a valuable fermentable sugar for xylitol production, as well as for repeated batch pretreatment of biomass. These strategies could be employed to reduce wastewater while also making use of byproducts.

\section{DATA AVAILABILITY}

The raw data supporting the conclusions of this manuscript will be made available by the authors, without undue reservation, to any qualified researcher.

\section{AUTHOR CONTRIBUTIONS}

SK conceived and designed the experiment, performed the experiment, analyzed the data and, wrote the manuscript. 


\section{ACKNOWLEDGMENTS}

This work was partially supported by Research Exchange Program under Memorandum of Understanding between the NRF-DAAD (NRF-2013K2A5A5079255) from Korea National Research Foundation (NRF), the New \&

\section{REFERENCES}

Abdolali, A., Guo, W. S., Ngo, H. H., Chen, S. S., Nguyen, N. C., and Tung, K. L. (2014). Typical lignocellulosic wastes and by-products for biosorption process in water and wastewater treatment: a critical review. Bioresour. Technol. 160, 57-66. doi: 10.1016/j.biortech.2013.12.037

Aliakbarian, B., de Faveri, D., Perego, P., and Converti, A. (2012). "An assessment on xylitol recovery methods," in D-xylitol, eds S. S. da Silva and A. K. Chandel (Berlin; Heidelberg: Springer-Verlag), 229-244. doi: 10.1007/978-3-642-31887-0_10

Alvira, P., Tomás-Pejó, E., Ballesteros, M., and Negro, M. J. (2010). Pretreatment technologies for an efficient bioethanol production process based on enzymatic hydrolysis: a review. Bioresour. Technol. 101, 4851-4861. doi: 10.1016/j.biortech.2009.11.093

Brethauer, S., and Studer, M. H. (2015). Biochemical conversion processes of lignocellulosic biomass to fuels and chemicals-a review. Chimia 69, 572-581. doi: 10.2533/chimia.2015.572

Caicedo, M., Barros, J., and Ordás, B. (2016). Redefining agricultural residues as bioenergy feedstocks. Materials 9:E635. doi: 10.3390/ma9080635

Capolupo, L., and Faraco, V. (2016). Green methods of lignocellulose pretreatment for biorefinery development. Appl. Microbiol. Biotechnol. 100, 9451-9467. doi: 10.1007/s00253-016-7884-y

de Witt, R. N., Kroukamp, H., and Volschenk, H. (2019). Proteome response of two natural strains of Saccharomyces cerevisiae with divergent lignocellulosic inhibitor stress tolerance. FEMS Yeast Res. 19:foyl16. doi: 10.1093/femsyr/foy116

Humbird, D., Davis, R., Tao, L., Kinchin, C., Hsu, D., Aden, A., et al. (2011). Process Design and Economics for Biochemical Conversion of Lignocellulosic Biomass to Ethanol. Dilute-acid Pretreatment and Enzymatic Hydrolysis of Corn Stover, NREL/TP-5100-47764. Available online at: https://www.nrel.gov/docs/fy11osti/ 47764.pdf

Jönsson, L. J., and Martín, C. (2016). Pretreatment of lignocellulose: formation of inhibitory by-products and strategies for minimizing their effects. Bioresour. Technol. 199, 103-112. doi: 10.1016/j.biortech.2015.10.009

Kim, S. (2018a). Evaluation of alkali-pretreated soybean straw for lignocellulosic bioethanol production. Int. J. Polym. Sci. 2018:5241748. doi: $10.1155 / 2018 / 5241748$

Kim, S. (2018b). Enhancing bioethanol productivity using alkali-pretreated empty palm fruit bunch fiber hydrolysate. Biomed Res. Int. 2018:5272935. doi: $10.1155 / 2018 / 5272935$

Kim, S., and Kim, C. H. (2012). Production of cellulose enzymes during the solidstate fermentation of empty palm fruit bunch fiber. Bioproc. Biosyst. Eng. 35, 61-67. doi: 10.1007/s00449-011-0595-y

Kim, S., and Kim, C. H. (2013). Bioethanol production using the sequential acid/alkali-pretreated empty palm fruit bunch fiber. Renew. Energ. 54, 150-155. doi: 10.1016/j.renene.2012.08.032

Kim, S., and Kim, C. H. (2014). Evaluation of whole Jerusalem artichoke (Helianthus tuberosus L.) for consolidated bioprocessing ethanol production. Renew. Energ. 65, 83-91. doi: 10.1016/j.renene.2013.07.025

Kim, S., Lee, J., and Sung, B. H. (2019). Isolation and characterization of the stress-tolerant Candida tropicalis YHJ1 and evaluation of its xylose reductase for xylitol production from acid pretreatment wastewater. Front. Bioeng. Biotechnol. 7:138. doi: 10.3389/fbioe.2019.00138

Kim, S., Park, J. M., and Kim, C. H. (2013). Ethanol production using whole plant biomass of Jerusalem artichoke by Kluyveromycs marxianus CBS1555. Appl. Biochem. Biotechnol. 169, 1531-1545. doi: 10.1007/s12010-013-0094-5

Kim, S., Park, J. M., Seo, J. W., and Kim, C. H. (2012). Sequential acid-/alkalipretreatment of empty palm fruit bunch fiber. Bioresour. Technol. 109, 229-233. doi: 10.1016/j.biortech.2012.01.036
Renewable Energy Technology Development Program of the Korea Institute of Energy Technology Evaluation and Panning (KETEP) granted financial resource from the Ministry of Trade, Industry \& Energy, Republic of Korea (No. 20133010091700) and KRIBB Research Initiative

Program grant.
Liu, W., Wang, J., Richard, T. L., Hartley, D. S., Spatari, S., and Volk, T. A. (2017). Economic and life cycle assessments of biomass utilization for bioenergy products. Biofuels Bioprod. Bioresour. 11, 633-647. doi: 10.1002/bbb. 1770

Liu, Z. L. (2011). Molecular mechanisms of yeast tolerance and in situ detoxification of lignocellulose hydrolysates. Appl. Microbiol. Biotechnol. 90, 809-825. doi: 10.1007/s00253-011-3167-9

Melin, K., and Hurme, M. (2011). Lignocellulosic biorefinery economic evaluation. Cellulose Chem. Technol. 45, 443-454.

Mpabanga, T. P., Chandel, A. K., da Silva, S. S., and Singh, O. V. (2012). "Detoxification strategies applied to lignocellulosic hydrolysates for improved xylitol production," in D-xylitol, eds S. S. da Silva and A. K. Chandel (Berlin; Heidelberg: Springer-Verlag), 63-82.

Palmqvist, E., and Hahn-Hägerdal, B. (2000). Fermentation of lignocellulosic hydrolysates. II: inhibitors and mechanisms of inhibition. Bioresour. Technol. 74, 25-33. doi: 10.1016/S0960-8524(99)00161-3

Parajó, J. C., Domínguez, H., and Domínguez, J. M. (1997). Xylitol production from Eucalyptus wood hydrolysates extracted with organic solvents. Process Biochem. 32, 599-604. doi: 10.1016/S0032-9592(97)00 016-2

Parajó, J. C., Domínguez, H., and Domínguez, J. M. (1998). Biotechnological production of xylitol. Part 3: operation in culture meida made from lignocellulose hydrolysates. Bioresour. Technol. 66, 25-40. doi: 10.1016/S0960-8524(98)00037-6

Piarpuzán, D., Quintero, J. A., and Cardona, C. A. (2011). Empty fruit bunches from oil palm as a potential raw material for fuel ethanol production. Biomass Bioenerg. 35, 1130-1137. doi: 10.1016/j.biombioe.2010. 11.038

Raman, J. K., and Gnansounou, E. (2014). Ethanol and lignin production from Brazilian empty fruit bunch biomass. Bioresour. Technol. 172, 241-248. doi: 10.1016/j.biortech.2014.09.043

Ravella, S. R., Gallagher, J., Fish, S., and Prakasham, R. S. (2012). "Overview on commercial production of xylitol, economic analysis and market trends," in D-xylitol, eds S. S. da Silva and A. K. Chandel (Berlin; Heidelberg: SpringerVerlag), 291-306.

Shen, Y., Jarboe, L., Brown, R., and Wen, Z. (2015). A thermochemicalbiochemical hybrid processing of lignocellulosic biomass for producing fuels and chemicals. Biotechnol. Adv. 33, 1799-1813. doi: 10.1016/j.biotechadv.2015. 10.006

Silveira, M. H., Morais, A. R., da Costa Lopes, A. M., Olekszyszen, D. N., BogelŁukasik, R., Andreaus, J., et al. (2015). Current pretreatment technologies for the development of cellulosic ethanol and biorefineries. ChemSusChem 8 , 3366-3390. doi: 10.1002/cssc.201500282

Thompson, O. A., Hawkins, G. M., Gorsich, S. W., and Doran-Peterson, J. (2016). Phenotypic characterization and comparative transcriptomics of evolved Saccharomyces cerevisiae strains with improved tolerance to lignocellulosic derived inhibitors. Biotechnol. Biofuels 9:200. doi: 10.1186/s13068-016-0614-y

Toivari, M. H., Nygård, Y., Penttilä, M., Ruohonen, L., and Wiebe, M. G. (2012). Microbial D-xylonate production. Appl. Microbiol. Biotechnol. 96, 1-8. doi: $10.1007 / \mathrm{s} 00253-012-4288-5$

Vinke, P., and van Bekkum, H. (1992). The dehydration of fructose towards 5 Hydroxymethylfurfural using activated carbon as adsorbent. Starch 40, 90-96. doi: 10.1002/star.19920440303

Wang, S., Li, H., Fan, X., Zhang, J., Tang, P., and Yuan, Q. (2015). Metabolic responses in Candida tropicalis to complex inhibitors during xylitol bioconversion. Fungal Genet. Biol. 82, 1-8. doi: 10.1016/j.fgb.2015 04.022

Werpy, T., Peterson, G., Aden, A., Bozell, L., Holladay, J., White, J., et al. (2004) Top Value Added Chemicals from Biomass, Vol I: Results of Screening for 
Potential Candidates from Sugars and Synthesis Gas. US Department of Energy. Available online at: https://www.nrel.gov/docs/fy04osti/35523.pdf

Zhang, K., Agrawal, M., Harper, J., Chen, R., and Koros, W. J. (2011). Removal of the fermentation inhibitor, furfural, using activated carbon in cellulosicethanol production. Ind. Eng. Chem. Res. 50, 14055-14060. doi: 10.1021/ ie 2013983

Zhang, Q. H., Lu, X., Tang, L., Mao, Z. G., Zhang, J. H., Zhang, H. J., et al. (2010). A novel full recycling process through two-stage anaerobic treatment of distillery wastewater for bioethanol production from cassava. J. Hazard. Mater. 179, 635-641. doi: 10.1016/j.jhazmat.2010.03.050
Conflict of Interest Statement: The author declares that the research was conducted in the absence of any commercial or financial relationships that could be construed as a potential conflict of interest.

Copyright $\odot 2019 \mathrm{Kim}$. This is an open-access article distributed under the terms of the Creative Commons Attribution License (CC BY). The use, distribution or reproduction in other forums is permitted, provided the original author(s) and the copyright owner(s) are credited and that the original publication in this journal is cited, in accordance with accepted academic practice. No use, distribution or reproduction is permitted which does not comply with these terms. 